\title{
A CHARACTERIZATION OF 2-DIMENSIONAL SPHERICAL SPACE
}

\author{
L. D. LOVELAND AND J. E. VALENTINE
}

Abstract. The midset of two distinct points $a$ and $b$ of a metric space is defined as the set of all points $x$ in the space for which the distances $a x$ and $b x$ are equal. A metric space is said to have the 1-WLMP if the midset of each two distinct points is a convex 1sphere having the property that each nonmaximal (with respect to inclusion) segment intersecting it twice lies in it. We show that a nondegenerate compact space $X$ is isometric to a 2-dimensional spherical space $S_{2, \alpha}$ (a 2-dimensional sphere of radius $\alpha$ in euclidean 3-space with the "shorter arc" metric) if and only if $X$ has a metric with the 1-WLMP.

Berard ([1], [2]) has given characterizations of both the 1-sphere and the 1-cell using conditions on the midsets of points in a metric space, and Buseman [4] characterized euclidean, hyperbolic, and spherical spaces among his $G$-spaces using convex midset properties. We characterize 2-dimensional spherical space among nontrivial compact metric spaces using a certain linear midset property described below.

The midset $M(a, b)$ of two distinct points $a$ and $b$ of a metric space $X$ is the set of all points $x$ in $X$ for which the distances $a x$ and $b x$ are equal. A metric space $X$ is said to have the weak linear midset property (WLMP) if, for each two distinct points $a$ and $b$ of $X$, the midset $M(a, b)$ contains every nonmaximal segment (with respect to inclusion) that intersects it twice. If in addition to having the WLMP each midset in $X$ is a convex 1 -sphere we say that $X$ has the 1-WLMP.

We prove that a nontrivial compact metric space $X$ with the 1-WLMP is isometric to a 2-dimensional spherical space. The proof of this result is delayed until after a sequence of lemmas has been given. In each of these lemmas it is to be understood that $X$ is a nontrivial (nondegenerate) compact metric space with the 1-WLMP. The symbol $S(a, b)$ is used to denote a metric segment with endpoints $a$ and $b$, and the fact that $q$ is between the points $p$ and $r$ (that is, $p \neq q \neq r$ and $p q+q r=p r$ ) will be denoted by writing pqr. A segment $S$ is said to be maximal if it is not a proper subset of another segment. In a compact convex metric space it is well known that each two distinct points are the endpoints of at least one

Received by the editors June 20, 1972.

AMS (MOS) subject classifications (1970). Primary 52A50.

(c) American Mathematical Society 1973 
segment [3, p. 41], and it is easy to show that each segment lies in a maximal one.

LEMMA 1. If $a$ and $b$ are distinct points of $X$, then there exist points $p$ and $q$ in $M(a, b)$ and a segment $S(a, b)$ in $X$ such that $S(a, b)$ lies in $M(p, q)$. In particular it follows that $X$ is convex.

Proof. Let $f$ denote a foot of $a$ on $M(a, b)$. Since $M(a, b)$ is a convex simple closed curve it contains two segments $S(c, f)$ and $S(d, f)$ whose intersection is $\{f\}$. We may assume that $a c \leqq a d$, and it follows that $a f \leqq a c \leqq a d$. If equality holds in one of these inequalities, then $a$ and $b$ lie in a midset. This midset would contain a segment $S(a, b)$ since it is complete and convex. In the other case where $a f<a c<a d$, the continuous function $a x$, with $x$ in $S(f, d)$, assumes the value $a c$ at some point $q$ in $S(f, d)$. If we let $c=p$, we have $a p=a q=b q=b p$, and we see that $a$ and $b$ are in the midset of $p$ and $q$. As before, $M(p, q)$ contains a segment $S(a, b)$.

LEMMA 2. If $a$ and $b$ are distinct points of $X$ and $S(a, b)$ is a nonmaximal segment, then $S(a, b)$ is the unique segment in $X$ having endpoints $a$ and $b$.

Proof. Let $S(a, b)$ and $S_{1}(a, b)$ be two distinct segments, and suppose that $S(a, b)$ is properly contained in a segment $S$. We choose points $x$ and $y$ in $S_{1}(a, b) \cap S(a, b)$ such that the subsegments $S_{1}^{\prime}(x, y)$ and $S^{\prime}(x, y)$ of $S_{1}(a, b)$ and $S(a, b)$, respectively, intersect only at their endpoints. Let $m_{1}$ and $m$ be the midpoints of $S_{1}^{\prime}(x, y)$ and $S^{\prime}(x, y)$, respectively. Clearly the midset $M\left(m_{1}, m\right)$ contains both $x$ and $y$. Therefore it follows from the WLMP that $M\left(m_{1}, m\right)$ contains $S^{\prime}(x, y)$, which contradicts the fact that $m_{1} m_{1} \neq m_{1} m$.

A point $q$ is called a ramification point of a metric space $X$ if there exist pairwise distinct points $p, r$, and $r^{\prime}$ of $X$ such that $q$ is a midpoint of $p$ and $r$ and $q$ is a midpoint of $p$ and $r^{\prime}$. If $X$ is compact and convex and if $X$ has a ramification point $q$, it follows that there exist two segments $S(p, r)$ and $S\left(p, r^{\prime}\right)$ both having $q$ as a midpoint [3, p. 44].

LEMMA 3. The space $X$ has no ramification points.

Proof. Suppose that $X$ has a ramification point $q$, and let $S(p, r)$ and $S\left(p, r^{\prime}\right)$ be two distinct segments with $q$ as their midpoint. Select points $x$ and $y$ in the interiors of $S(p, r)$ and $S\left(p, r^{\prime}\right)$, respectively, such that $q x r, q y r$, and $q x=q y$. Now both $p$ and $q$ lie in $M(x, y)$; hence it follows from the WLMP that $x$ and $y$ belong to $M(x, y)$. This contradiction establishes the lemma.

LEMMA 4. If $a$ and $b$ are distinct points of $X$, then $M(a, b)$ separates $a$ from $b$ in $X$. In fact, $X-M(a, b)=A \cup B$, where $A=\{y \in X \mid a y<b y\}$ and $B=\{y \in \mid X a y>b y\}$, is the desired separation. 
LeMMA 5. If $a$ and $b$ are distinct points of $X$ and $u$ is a point in $M(a, b)$, then $M(a, b)$ contains $a$ point $v$ and two maximal segments whose union is $M(a, b)$ and whose intersection is $\{u, v\}$.

Proof. Since $M(a, b)$ is a simple closed curve it contains distinct points $u$ and $t$. Since $M(a, b)$ is compact and convex, it contains a segment $S(u, t)$. Thus the partially ordered collection of all segments $S(u, x)$ (ordered by inclusion) with one endpoint $u$, containing $S(u, t)$, and lying in $M(a, b)$ has a maximal element which we call $S_{1}(u, v)$.

Letting $\left\{x_{i}\right\}$ be a sequence of points in $M(a, b)-S_{1}(u, v)$ converging to $v$, we see that $M(a, b)$ contains a segment $S\left(u, x_{n}\right)$ such that $S\left(u, x_{n}\right)$ and $S_{1}(u, v)$ have only the point $u$ in common. A positive integer $N$ exists such that $u x_{1} x_{n}$ holds for $n>N$; thus $u x_{1}+x_{1} x_{n}=u x_{n}$. By the continuity of the metric it follows that $u x_{1} v$ holds, and since $M(a, b)$ is compact and convex, it contains two segments $S\left(u, x_{1}\right)$ and $S\left(x_{1}, v\right)$ whose union is a segment $S_{2}(u, v)$ [3, p. 44]. It is clear that $S_{1}(u, v) \cup S_{2}(u, v)=M(a, b)$ since $M(a, b)$ is a simple closed curve, and from the construction of $S_{2}(u, v)$ we see that $S_{1}(u, v) \cap S_{2}(u, v)=\{u, v\}$. If $S_{2}(u, v)$ were not maximal it would follow from the WLMP that $M(a, b)$ would contain a point $e$ such that either uve or vue holds, contrary to the fact that uev holds since $e$ would lie in $S_{1}(u, v)$.

Definition. Let $a$ and $b$ be distinct points of $X$. The cone on $M(a, b)$ from $a$ is the union of all segments $S(a, y)$ where $y$ lies in $M(a, b)$.

LeMma 6. Let $a$ and $b$ be distinct points of $X$. If for each point $y$ in $M(a, b)$ there is $a$ unique segment with endpoints $a$ and $y$, then the cone on $M(a, b)$ from a is a 2-cell.

Proof. We first note that if $x$ and $y$ are distinct points of $M(a, b)$, then $S(a, x)$ and $S(a, y)$ have only the point $a$ in common. Otherwise $X$ would either contain a ramification point (contrary to Lemma 3 ) or the WLMP would imply that $a$ lies in $M(a, b)$ (contrary to $a a \neq a b$ ).

Let $S$ denote the circle $\left\{(x, y, 0) \mid x^{2}+y^{2}=1\right\}$ in $E^{3}$, and let $f$ be a homeomorphism from $M(a, b)$ onto $S$. We denote $f(y)$ by $y^{\prime}$, and we define $a^{\prime}=f(a)$ to be the point $(0,0,1)$ in $E^{3}$. We extend $f$ to a homeomorphism from the cone $C$ on $M(a, b)$ from $a$ onto the cone $C^{\prime}$ on $S$ from $a^{\prime}$ as follows. For each $x$ in $C-M(a, b)-\{a\}$ there is a unique point $y$ such that axy holds. Let $x^{\prime}=f(x)$ be that point on $S\left(a^{\prime}, y^{\prime}\right)$ such that $a^{\prime} x^{\prime} \mid a^{\prime} y^{\prime}=$ ax/ay. From above it is clear that $f$ is a bijection. If $\left\{x_{n}\right\}$ is a sequence of points of $C$ converging to $x_{0}$, and, for each $i, y_{i}$ is the point of $M(a, b)$ such that $x_{i}$ belongs to $S\left(a, y_{i}\right)$, then it follows that $\left\{y_{n}\right\}$ converges to $y_{0}$. Thus $\left\{y_{n}^{\prime}\right\}$ converges to $y_{0}^{\prime}$, and then $\left\{x_{n}^{\prime}\right\}$ must converge to $x_{0}^{\prime}$. In the same 
manner we see that $f^{-1}$ is continuous; hence $f$ is a homeomorphism. Since $C^{\prime}$ is known to be a 2-cell, the lemma follows.

THEOREM 1. A nondegenerate compact space $X$ is a 2-sphere if and only if there is a metric for $X$ under which $X$ has the WLMP.

Proof. If $X$ is a 2-sphere, then it is homeomorphic to

$$
\left\{(x, y, z) \mid x^{2}+y^{2}+z^{2}=1\right\}
$$

in $E^{3}$. The usual "shortest arc" metric on this round sphere satisfies the conditions of the theorem. For the other half of the proof we assume that $X$ is a nondegenerate compact metric space with the 1-WLMP.

Let $a^{\prime}$ and $b^{\prime}$ be two distinct points of $X$, and let $u$ be in $M\left(a^{\prime}, b^{\prime}\right)$. According to Lemma 5 there exists another point $v$ in $M\left(a^{\prime}, b^{\prime}\right)$ and two maximal segments whose union is $M\left(a^{\prime}, b^{\prime}\right)$ and whose intersection is $\{u, v\}$. If $m$ and $m^{\prime}$ are the midpoints of these maximal segments, then $m$ and $m^{\prime}$ belong to the convex compact set $M(u, v)$. Then $M(u, v)$ contains a segment $S\left(m, m^{\prime}\right)$, and this segment is maximal for otherwise either $u$ or $v$ would belong to $M(u, v)$ by the WLMP. From Lemma 5 there is another maximal segment in $M(u, v)$ joining $m$ to $m^{\prime}$. We let $a$ and $b$ be the midpoints of these two maximal segments in $M(u, v)$. Note that $m$ and $m^{\prime}$ belong to $M(a, b)$, and since $a$ and $b$ lie in $M(u, v)$ we know that $u a=a v$ and $b u=b v$. Now $a, b, u$, and $v$ all lie in $M\left(m, m^{\prime}\right)$, and from the WLMP we see that any segment in $M\left(m, m^{\prime}\right)$ joining $u$ and $v$ is maximal. Since two such maximal segments exist in $M\left(m, m^{\prime}\right)$ we know that $u a v$ and $u b v$ hold. Thus $u a+a v=u v=u b+b v$ and it follows that $2(a u)=u v$ and $2(b u)=$ $u v$. Thus $a u=b u$, and similarly $a v=b v$. This means that $u$ and $v$ belong to $M(a, b)$. Since $m, m^{\prime}, u$, and $v$ all lie in $M(a, b)$, it follows from the WLMP that $M(a, b)=M\left(a^{\prime}, b^{\prime}\right)$. We have no more need for $a^{\prime}$ and $b^{\prime}$.

We now show that for each $y$ in $M(a, b)$ there is a unique segment with endpoints $a$ and $y$. If $y$ is either $m$ or $m^{\prime}$, then a nonmaximal segment $S(a, y)$ exists in $M(u, v)$, and therefore $S(a, y)$ is unique by Lemma 2 . Since $M\left(m, m^{\prime}\right)$ contains $a, b, u$, and $v$ it follows from the WLMP that there is a nonmaximal segment $S(a, y)$ in $M\left(m, m^{\prime}\right)$ if $y$ is either $u$ or $v$. Thus such a segment is also unique. We now assume that $y \notin\left\{m, m^{\prime}, u, v\right\}$. By relabeling points if necessary it may be assumed that $m y u$ holds. From Lemma 1 we know that there exists a segment $S(a, y)$ and two distinct points $p$ and $q$ in $M(a, y)$ such that $M(p, q)$ contains $S(a, y)$. Since the segment $S(a, y)$ is known to be unique unless it is a maximal segment (by Lemma 2), we suppose that $S(a, y)$ is maximal in order to obtain a contradiction. It follows from Lemma 5 that $M(p, q)$ is the union of two maximal segments $S_{1}(a, y)$ and $S(a, y)$ whose intersection is $\{a, y\}$. Notice that $M(p, q) \cap M(a, b)=\{y\}$, for otherwise the 1-WLMP implies 
the contradiction that $a$ belongs to $M(a, b)$. Let $r$ and $n$ be the midpoints of $S(a, y)$ and $S_{1}(a, y)$, respectively, and note that $M(a, y)$ contains $\{r, n\}$. Thus, from Lemma $5, M(a, y)$ is the union of two segments $S_{1}$ and $S_{2}$, having endpoints $r$ and $n$, such that $S_{1} \cap S_{2}=\{r, n\}$. Let $A$ and $B$ be the mutually separated sets promised by Lemma 4 whose union is $X-M(a, b)$ with $a \in A$ and $b \in B$. Notice that both $r$ and $n$ belong to $A$. Suppose that there is a point $f$ of $B$ in one of the two segments $S_{1}$ and $S_{2}$. Then $M(a, b)$ would contain two interior points of $S_{i}, i=1$ or 2 , since the endpoints of $S_{i}$ both lie in $A$. From WLMP it would follow that $f$ lies in $M(a, b)$, a contradiction. Thus $M(a, y) \cap B=\varnothing$. Since $M(a, y)$ separates $a$ from $y$ it must follow that $M(a, y)$ intersects each of the unique segments $S(a, u), S(a, m), S\left(a, m^{\prime}\right)$, and $S(a, v)$. Since $S(a, u) \cup$ $S(a, v)$ is a maximal segment in $M\left(m, m^{\prime}\right)$ (see Lemma 5 and the WLMP), $M(a, y)$ cannot intersect its interior twice. Then $u$ and $v$ must lie in $M(a, y)$. Similar reasoning shows that both $m$ and $m^{\prime}$ lie in $M(a, y)$. From the 1-WLMP it follows that $M(a, b)$ lies in $M(a, y)$, contrary to the fact that $y$ does not belong to $M(a, y)$. Therefore the segment $S(a, y)$ is unique. Similarly segments $S(b, y)$, with $y$ in $M(a, b)$, are unique.

Now we may apply Lemma 6 to obtain two 2-cells $D_{1}$ and $D_{2}$ by coning $M(a, b)$ from $a$ and $b$, respectively. We shall prove that the 2 -sphere $X^{\prime}=D_{1} \cup D_{2}$ is $X$. Suppose to the contrary that there is a point $y$ in $X-X^{\prime}$. We may suppose that $a y<b y$ since $y \notin M(a, b)$. Let $S(a, y)$ be a segment and notice that it does not intersect $M(a, b)$. Since $X$ has no ramification points (Lemma 3) it follows that $S(a, y) \cap X^{\prime}=\{a\}$. Choose a point $z$ in $M(a, b)$, and a segment $S(y, z)$. Order $S(y, z)$ from $y$ to $z$ and pick the first point $y^{\prime}$ of $X^{\prime} \cap S(y, z)$. Since $a y<b y$ and $S\left(y, y^{\prime}\right) \cap M(a, b)$ contains at most the point $y^{\prime}$, it follows that $y^{\prime}$ lies in $D_{1}$. Let $p$ be the point of $M(a, b)$ such that $y^{\prime} \in S(a, p)$. Now $M\left(a, y^{\prime}\right)$ cannot intersect the segment $S(a, p)$ at the point other than the midpoint $t$ of $S\left(a, y^{\prime}\right)$ by the WLMP. Thus there is a segment $S(h, k)$ in $M(a, b)$, with $p$ in its interior, such that $S(h, k) \cap M\left(a, y^{\prime}\right)=\varnothing$. The cone $D$ on $S(h, k)$ from $a$ is a 2-cell (see the proof of Lemma 6) in $D_{1}$. Each segment $S(a, x)$, with $x$ in $S(h, k)$, must intersect $M\left(a, y^{\prime}\right)$, for otherwise $M\left(a, y^{\prime}\right)$ would not separate $a$ from $y^{\prime}$ in $D$. From WLMP the intersection of $S(a, x)$ with $M\left(a, y^{\prime}\right)$ consists of a single point $w_{x}$. Let $R$ be the union of all segments $S\left(a, w_{x}\right), x$ in $S(h, k)$, and let $T$ be the union of all segments $S\left(w_{x}, x\right)$. Then $R \cup T=D$ and $R$ and $T$ are each closed and connected. From the unicoherence of $D$ [5, p. 374], $R \cap T$ is connected. Since $R \cap T \subset M\left(a, y^{\prime}\right)$, there must be an arc $G$ in $R \cap T$ with $t$ in its interior.

Let $\left\{x_{i}\right\}$ be a sequence of points in $S\left(y, y^{\prime}\right)$ converging to $y^{\prime}$, and notice that there is an integer $K$ such that, for $i>K$, each segment $S\left(a, x_{i}\right)$ intersects $M\left(a, y^{\prime}\right)$ at a unique point $t_{i}$. This is because $M\left(a, y^{\prime}\right)$ separates 
$a$ from $y^{\prime}$ in the simple closed curve in $S\left(a, x_{i}\right) \cup S\left(x_{i}, y^{\prime}\right) \cup S\left(a, y^{\prime}\right)$ and, for large $i, S\left(x_{i}, y^{\prime}\right) \cap M\left(a, y^{\prime}\right)=\varnothing$; the uniqueness of $t_{i}$ comes from the WLMP. The continuity of the metric insures that $\left\{t_{i}\right\}$ converges to $t$. This is a contradiction since it is impossible for the simple closed curve $M\left(a, y^{\prime}\right)$ to contain an arc $G$ and to contain a sequence $\left\{t_{i}\right\}$ of points not in $G$ but converging to the interior point $t$ of $G$.

This establishes the fact that $X$ is the 2-sphere $X^{\prime}$, and completes the proof of Theorem 1.

THEOREM 2. A nontrivial compact space $X$ is isometric to a 2-dimensional spherical space if and only if $X$ has a metric with the 1-WLMP.

Proof. Since 2-dimensional spherical space has a metric with the 1-WLMP we need only show the proof in the other direction. Thus we now assume that $X$ is a nontrivial compact metric space with the 1-WLMP. From Theorem 1, $X$ is homeomorphic to a 2 -sphere.

Busemann [4] has shown that a 2-dimensional compact $G$-space with convex midsets is isometric with 2-dimensional spherical space. Since $X$ is 2-dimensional, compact, and has convex midsets, Theorem 2 will follow from [4] once we show that $X$ is a $G$-space. The only condition on a $G$ space that is not either obvious or given by previous lemmas is the locally externally convex property. We shall now show that $X$ has this property.

We assume all of the proof and notation from Theorem 1 up to the last two paragraphs, so that we know the 2-sphere $X$ is the union of the two 2-cells $D_{1}$ and $D_{2}$. These cells are the cones from $a$ and $b$, respectively, on $M(a, b)$. A point $p$ of $X$ cannot lie in all three midsets $M(a, b), M(u, v)$, and $M(m, m)$, so we assume for convenience that $p$ is not in $M(a, b)$. A connected neighborhood $N$ of $p$ is chosen so that $N \cap M(a, b)=\varnothing$. Let $x$ and $y$ be two points of $N$. To show that $X$ is locally externally convex at $p$ it suffices to exhibit a point $z$ in $N$ such that $x y z$. From Lemma 1 we see that there exist two points $q$ and $r$ and a segment $S$ joining $x$ and $y$ such that $S \subset M(q, r)$. The selection of $z$ can be made in $M(q, r)$ if $S$ is not a maximal segment. Suppose that $S$ is a maximal segment in the simple closed curve $M(q, r)$. Since $N$ does not intersect $M(a, b)$, we may assume that $x$ and $y$ are both closer to $a$ than to $b$. From the WLMP it follows that $S$ lies in $D_{1}$. (Notice that if we had assumed that $p$ was not in some midset other than $M(a, b)$ at the outset, then we could still go through the proof of Theorem 1 to write $X$ as the union of two 2-cells $D_{1}^{\prime}$ and $D_{1}^{\prime}$, each a cone on a midset. Thus the proof would go through just the same.) Since $M(q, r)$ is the union of two maximal segments $S$ and $S^{\prime}$ with endpoints $x$ and $y$ and since $S$ lies in $D_{1}$, it follows from the WLMP that $M(q, r)$ lies in $D_{1}$. Unless $M(q, r)$ separates $a$ from $M(a, b)$, some segment from $a$ to $M(a, b)$ would intersect $M(q, r)$ twice and would consequently 
lie in $M(q, r)$. This would force $N$ to contain a point of $M(a, b)$. Thus $M(q, r)$ separates $a$ from $M(a, b)$. But then a segment $S\left(m, m^{\prime}\right)$ in $M(u, v)$ would intersect $M(q, r)$ twice (at least once in its interior) and would lie in $M(q, r)$ by the WLMP. Again this would contradict the fact that $N$ does not intersect $M(a, b)$.

Thus $X$ is locally externally convex and the proof is complete.

\section{REFERENCES}

1. Anthony D. Berard, Jr., Characterizations of metric spaces by the use of their midsets: Intervals, Fund. Math. 73 (1971), 1-7.

2. - Characterizations of metric spaces by the use of their midsets: One spheres, Fund. Math. (to appear).

3. Leonard M. Blumenthal, Theory and applications of distance geometry, Clarendon Press, Oxford, 1953. MR 14, 1009.

4. H. Busemann, The geometry of geodesics, Academic Press, New York, 1955. MR 17,779 .

5. K. Kuratowski, Topologie. Vol. II, 3rd ed., Monografie Mat., Tom 21, PWN, Warsaw, 1961; English transl., Academic Press, New York; PWN, Warsaw, 1968. MR 24 \#A2958; MR 41 \#4467.

Department of Mathematics, Utah State University, Logan, Utah 84321 